\title{
Fab antibody fragment-functionalized liposomes for specific targeting of antigen-positive cells
}

Anna Ohradanova-Repic, $\mathrm{PhD}^{\mathrm{a}, *}$, Eugénia Nogueira, $\mathrm{PhD}^{\mathrm{b}, \mathrm{c}}$, Ingrid Hartl, $\mathrm{MSc}^{\mathrm{a}}$, Andreia C. Gomes, $\mathrm{PhD}^{\mathrm{c}}$, Ana Preto, $\mathrm{PhD}^{\mathrm{c}}$, Eva Steinhuber ${ }^{\mathrm{a}}$, Vanessa Mühlgrabner, $\mathrm{MSc}^{\mathrm{a}}$, Marko Repic, $\mathrm{Dr}^{\mathrm{d}}$, Mario Kuttke, $\mathrm{PhD}^{\mathrm{e}}$, Alexander Zwirzitz, $\mathrm{PhD}^{\mathrm{a}}$, Marek Prouza, $\mathrm{PhD}^{\mathrm{f}}$, Miloslav Suchanek, $\mathrm{PhD}^{\mathrm{f}}$, Gordana Wozniak-Knopp, $\mathrm{Dr}^{\mathrm{g}}$, Vaclav Horejsi, $\mathrm{PhD}^{\mathrm{h}}$, Gernot Schabbauer, $\mathrm{Dr}^{\mathrm{e}}$, Artur Cavaco-Paulo, $\mathrm{PhD}^{\mathrm{b}}$, Hannes Stockinger, $\mathrm{PhD}^{\mathrm{a}}$

${ }^{\mathrm{a}}$ Institute for Hygiene and Applied Immunology, Center for Pathophysiology, Infectiology and Immunology, Medical University of Vienna, Vienna, Austria

${ }^{\mathrm{b}}$ Centre of Biological Engineering, University of Minho, Campus of Gualtar, Braga, Portugal

${ }^{\mathrm{c}}$ Centre of Molecular and Environmental Biology, Department of Biology, University of Minho, Campus of Gualtar, Braga, Portugal

${ }^{\mathrm{d}}$ MC Toxicology Consulting, Vienna, Austria

${ }^{\mathrm{e}}$ Institute for Vascular Biology and Thrombosis Research, Center for Physiology and Pharmacology, Medical University of Vienna, Vienna, Austria ${ }^{\mathrm{f}}$ EXBIO Praha, Vestec, Czech Republic

${ }^{g}$ Christian Doppler Laboratory for Innovative Immunotherapeutics, Department of Biotechnology, University of Natural Resources and Life Sciences (BOKU), Vienna, Austria

${ }^{\mathrm{h}}$ Institute of Molecular Genetics of the Academy of Sciences of the Czech Republic, Prague, Czech Republic

Received 13 February 2017; accepted 13 September 2017

\begin{abstract}
Liposomes functionalized with monoclonal antibodies or their antigen-binding fragments have attracted much attention as specific drug delivery devices for treatment of various diseases including cancer. The conjugation of antibodies to liposomes is usually achieved by covalent coupling using cross-linkers in a reaction that might adversely affect the characteristics of the final product. Here we present an alternative strategy for liposome functionalization: we created a recombinant Fab antibody fragment genetically fused on its C-terminus to the hydrophobic peptide derived from pulmonary surfactant protein $\mathrm{D}$, which became inserted into the liposomal bilayer during liposomal preparation and anchored the Fab onto the liposome surface. The Fab-conjugated liposomes specifically recognized antigen-positive cells and efficiently delivered their cargo, the Alexa Fluor 647 dye, into target cells in vitro and in vivo. In conclusion, our approach offers the potential for straightforward development of nanomedicines functionalized with an antibody of choice without the need of harmful cross-linkers.
\end{abstract}

(C) 2017 Elsevier Inc. All rights reserved.

Key words: Active targeting; Liposome functionalization; Immunoliposome; Antibody engineering; Recombinant Fab antibody fragment

Liposomes have gained substantial attention as drug carriers in the last three decades. These $\approx 50-200 \mathrm{~nm}$ phospholipid vesicles are longer retained in blood and accumulate in pathological sites (tumors, infarcted or inflamed tissues) due to the enhanced permeability and retention effect, ultimately leading to higher efficacy and lower systemic toxicity comparing

Disclosure: The authors declare the following competing financial interest(s): E.N., A.C.G., A.P. and A.C.P. filed for a patent to use the neck domain peptide in folate-targeted liposomes for specific drug delivery. M.R. is an employee of MC Toxicology Consulting. M.P. and M.S. are employees of EXBIO Praha.

Funding: This work has received funding from the European Union's Seventh Framework Program (FP7/2007-2013; grant agreement NMP4-LA-2009-228827 NANOFOL) and Horizon 2020 Research and Innovation Program (grant agreement No 683356 - FOLSMART), further from the Portuguese Foundation for Science and Technology under the scope of the strategic funding of UID/BIO/04469/2013 unit and COMPETE 2020 (POCI-01-0145-FEDER-006684) and BioTecNorte operation (NORTE-01-0145-FEDER-000004) funded by the European Regional Development Fund under the scope of Norte2020.

*Corresponding author at: Medical University of Vienna, Kinderspitalgasse 15, A-1090, Vienna, Austria.

E-mail address: anna.repic@meduniwien.ac.at (A. Ohradanova-Repic). 

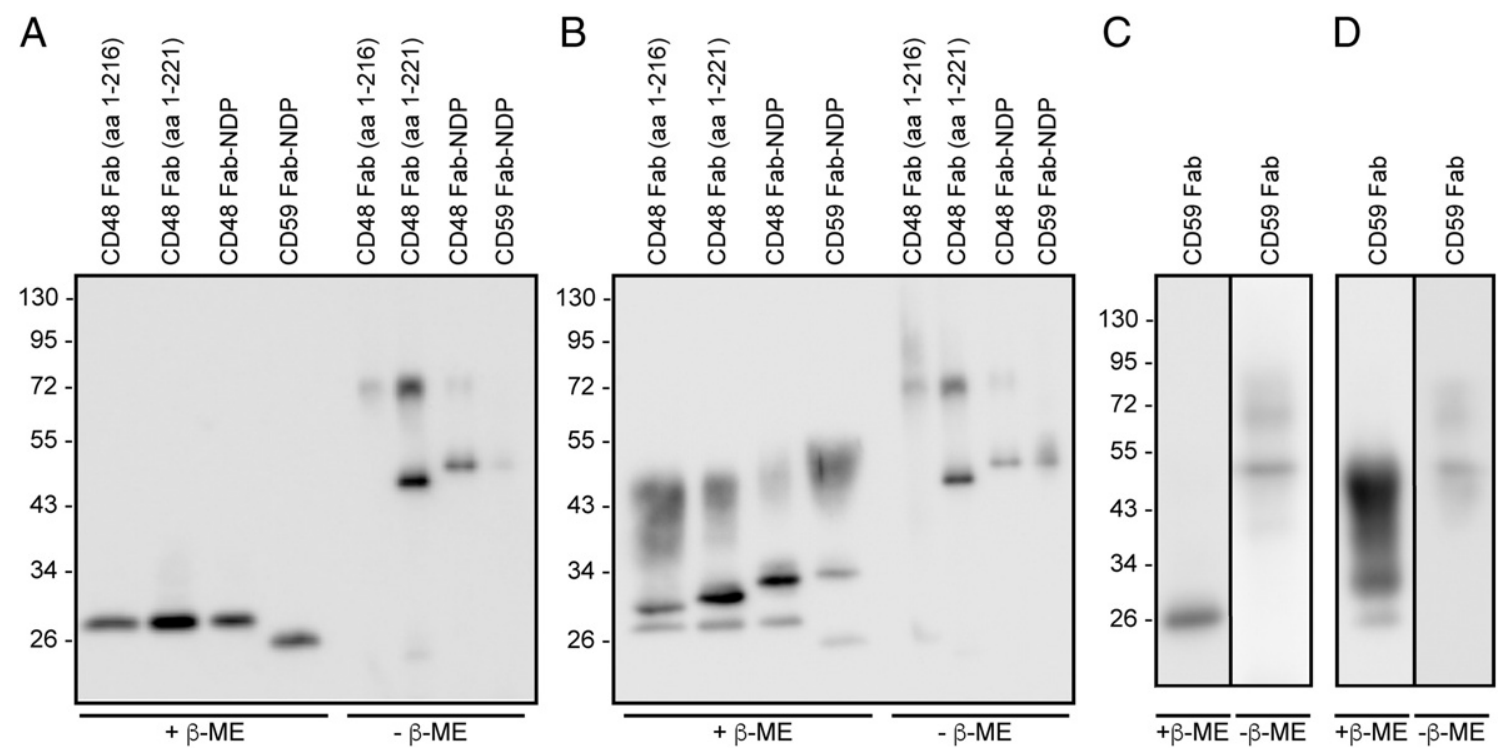

Figure 1. Analysis of the purified Fab fragments, either native or containing the neck domain peptide (NDP), by Western blotting. (A) The isolated CD48 and CD59 Fabs containing the NDP and two variants of the CD48 Fab were resolved by SDS-PAGE under reducing ( $+\beta-M E)$ or non-reducing $(-\beta-M E)$ conditions. The proteins were blotted, blocked and developed with HRP-labeled so-called anti-mouse IgG + IgM antibodies, which, however, recognized the light chains only as deduced from the analysis under reducing conditions in (A) and (B). (B) The membrane from (A) was reprobed with mouse anti-myc tag mAb (clone 9E10), recognizing the tagged Fab heavy chains, followed by the HRP-labeled anti-mouse antibodies. Similarly, the isolated CD59 Fab was resolved by SDS-PAGE and detected with HRP-conjugated anti-mouse antibodies only (C) or (D) with anti-myc tag mAb as in (B).

to the free drugs. Thanks to their amphiphilic nature, they transport well both hydrophobic and hydrophilic drugs. ${ }^{1-3}$ For these properties, liposomes are effective even as non-targeted devices and their efficacy is best demonstrated by liposomal doxorubicin Doxil ${ }^{\circledR}$, the first nano-drug approved for cancer treatment. ${ }^{4}$ Further improvement has been achieved with functionalization of liposomal surface for active targeting. The targeting ligands coupled to liposomal surface include proteins (monoclonal antibodies (mAbs) or their fragments), aptamers, vitamins, peptides or carbohydrates, which specifically recognize cell-surface proteins that are overexpressed on targeted cells. $^{2-4}$ Antibodies or antibody fragments - most commonly $\mathrm{scFv}$ and $\mathrm{Fab}$ - are frequently used due to their high specificity and affinity. They can be covalently coupled using various cross-linkers to either modified phospholipid head groups or onto the free terminus of poly(ethylene glycol) chains, which also serve as stealth. On the antibody side, the reactive groups of amino acid side chains (e.g. thiols and primary amines) are involved. Since antibodies may contain multiple reactive groups, the conjugation reaction could lead to heterogeneous $\mathrm{mAb}$-liposomes, where antibody is aggregated on the liposomal surface or is not properly oriented towards its antigen. ${ }^{2,5}$ In addition, covalent reactions require the need of chemical reagents that can potentially damage the targeting antibody or the liposome. ${ }^{6}$

For these reasons, we searched for an alternative way to functionalize liposomes with antibodies. We employed the hydrophobic peptide derived from the $\alpha$-helical neck domain of the pulmonary surfactant protein D (SP-D) that we genetically fused to the C-terminus of the Fab heavy chain. SP-D critically regulates ultrastructure of surfactant membranes ${ }^{7}$ and its neck domain shows affinity for phospholipids. ${ }^{8}$ Previously we showed that the SP-D neck domain peptide efficiently anchored folic acid into the liposomal membrane. ${ }^{9}$ Now we show that the same strategy can be used to equip liposomes with targeting proteins. We report here a full process of converting a mouse $\mathrm{mAb}$ into a recombinant $\mathrm{Fab}$ antibody fragment with $\mathrm{C}$-terminal neck domain peptide (NDP), its expression in the yeast Pichia pastoris and functionalization of liposomes with an isolated Fab-NDP construct. As a proof-of-concept, we targeted two well-characterized human glycosyl-phosphatidyl-inositol (GPI)-anchored cell-surface molecules, CD48 and CD59, with single-specific CD48 or CD59 Fab-functionalized liposomes as well as with double-specific liposomes containing both Fabs and demonstrated that the Fab-functionalized liposomes specifically targeted antigen-positive cells.

\section{Methods}

\section{Fab antibody fragment cloning and expression in P. pastoris}

The heavy and the light chains of the Fab antibody fragments from the CD48 mAb MEM-102 and the CD59 mAb MEM-43/5 were cloned from the respective mAb-producing hybridomas. The detailed cloning strategy, including tagging with the C-terminal NDP peptide, as well as Fab expression, purification and verification is provided in Supplementary data.

\section{Liposome preparation}

$\mathrm{pH}$-sensitive liposomes composed of DOPE1,2-Dioleoyl-snglycero-3-phosphoethanolamine)/CH (cholesterol)/DSPE-MPEG 


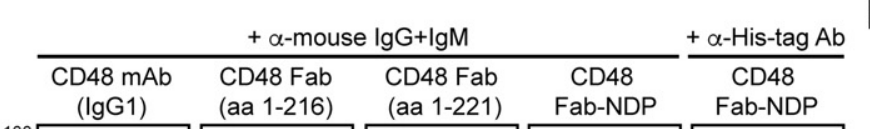

B
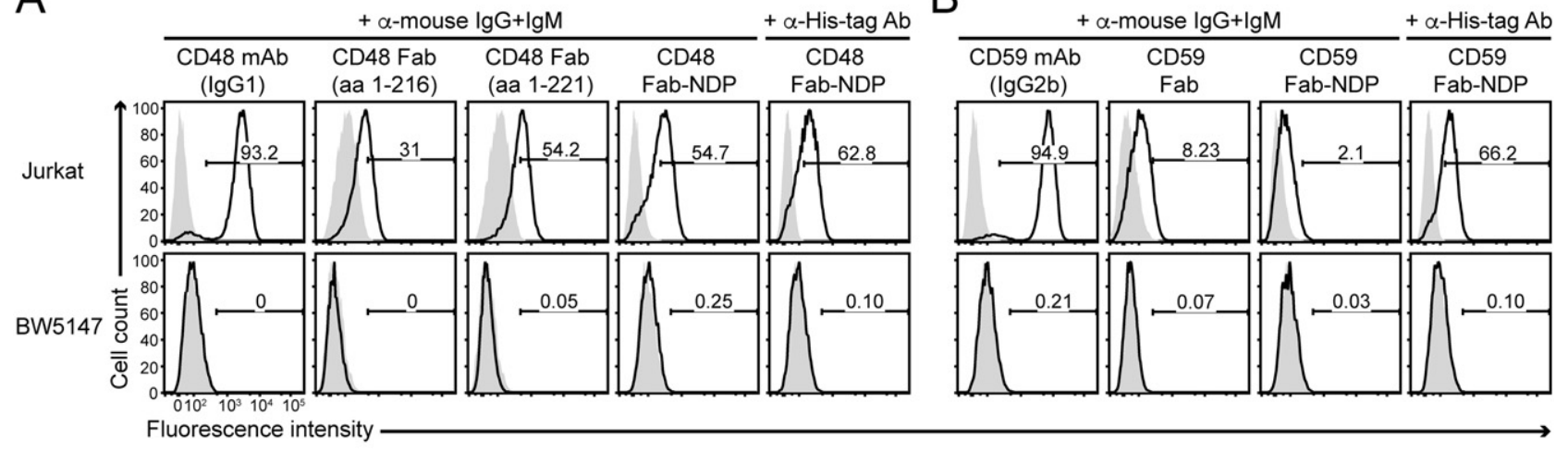

isotype control $\mathrm{mAb}$

specific staining as indicated

Figure 2. Functionality of the purified Fab fragments, either naked or containing the NDP, was tested by flow cytometry. (A) Cell surface staining of human CD48 Jurkat cells versus mouse BW5147 thymoma cells. Cells were stained with the parental CD48 mAb (IgG1) or recombinant CD48 Fabs (two naked forms of different lengths and the form containing the NDP) followed by visualization of binding using either APC-conjugated anti-mouse IgG + IgM antibodies or AF647-conjugated anti-His-tag mAb. The specific staining is overlaid by the background staining of the isotype control mAb (filled gray histograms). (B) Cell surface staining of CD59 $9^{+}$Jurkat cells versus mouse BW5147 cells. The binding of the CD59 Fab fragment containing the NDP was visualized as in (A). The parental CD59 mAb (IgG2b) served as positive control.

Table 1

The size, polydispersity index (PdI) and $\zeta$ potential measurements of liposomes.

\begin{tabular}{llll}
\hline Sample & $\begin{array}{l}\text { Z-average } \\
\text { size }[\mathrm{nm}]\end{array}$ & PdI & $\begin{array}{l}\text { 广 potential } \\
{[\mathrm{mV}]}\end{array}$ \\
\hline Liposomes & $128.0 \pm 2.4$ & 0.003 & -1.12 \\
CD48 Fab liposomes & $125.9 \pm 1.6$ & 0.015 & -1.76 \\
CD59 Fab liposomes & $126.8 \pm 1.8$ & 0.024 & -0.965 \\
CD48 + CD59 Fab liposomes & $127.0 \pm 2.0$ & 0.008 & -1.22 \\
\hline
\end{tabular}

([N-(carbonyl-methoxypolyethylene glycol-2000)-1,2-distearoylsn-glycero-3-phosphoethanolamine]) were prepared using a thin film hydration method as previously described. ${ }^{9,10}$ Briefly, DOPE, $\mathrm{CH}$ and DSPE-MPEG were dissolved in chloroform. After its evaporation, the dried lipid film was dispersed in PBS containing Fab-NDP antibody fragments and Alexa Fluor 647 (AF647). For creation of bispecific liposomes, the concentration of the CD48 and CD59 Fab-NDP fragments was 50\% reduced to keep the liposome loading constant. The mixture was vortex-mixed at $r$. t. to yield multilamellar vesicles, which were then extruded through 200 $\mathrm{nm}$ pore size polycarbonate filters (Nucleopore, Merck Millipore, Billerica, MA) followed by several passages through $100 \mathrm{~nm}$ polycarbonate filters (Nucleopore) to form large unilamellar vesicles. The free AF647 dye and Fabs that were not incorporated into liposomes were removed by gel filtration ( 5 kD cut-off column; GE Healthcare, Little Chalfont, UK) and dialysis (100 kD cut-off membrane; SERVA Electrophoresis, Heidelberg, Germany).

\section{Determination of $\zeta$ potential, polydispersity index and size}

Liposomes were characterized by the dynamic light scattering using a Malvern zetasizer NS (Malvern Instruments, Malvern, UK) as previously described. ${ }^{9}$
Further methods describing liposome specificity and toxicity tests are provided in Supplementary data.

\section{Statistics}

Statistical analysis and graphing was performed using Prism 5 (GraphPad Software, La Jolla, CA). Significance was accepted at $p<0.05$.

\section{Results}

\section{Fab antibody fragment cloning and expression}

The CD48 and CD59 molecules are GPI-anchored proteins that are localized in cholesterol-enriched membrane microdomains called lipid rafts. ${ }^{11}$ CD59 is broadly expressed and serves as an inhibitor of the cytolytic complement membrane attack complex, hence called protectin. ${ }^{12}$ In contrast, CD48, also known as BLAST-1, is rather restricted to immune cells, including lymphocytes and antigen presenting cells, and its expression is enhanced by inflammation. ${ }^{13}$ Both molecules have been shown to provide costimulatory signals during $\mathrm{T}$ cell activation. ${ }^{14-19}$ By employing mAbs specific for human CD48 (clone MEM-102) and human CD59 (clone MEM-43/5) we previously scrutinized molecular mechanisms how these GPI-anchored proteins were integrated into the $\mathrm{T}$ cell antigen receptor/CD3 signaling and early $\mathrm{T}$ cell activation events. ${ }^{14,16,18,20}$ In the present report, we used these mAbs as targeting agents to allow liposome functionalization and highly specific delivery of therapeutic drugs to CD48- and/or CD59-positive cells. As a first step, we created recombinant Fab antibody fragments of the aforementioned mAbs MEM-102 and MEM-43/5. For this, we isolated RNA from the respective $\mathrm{mAb}$-producing hybridoma cells, reverse transcribed it into cDNA and amplified VH-CH1 and VL-CL regions of the antibody chains by PCR. Amplification with a set of degenerate forward primers specific for the beginning of the Framework 


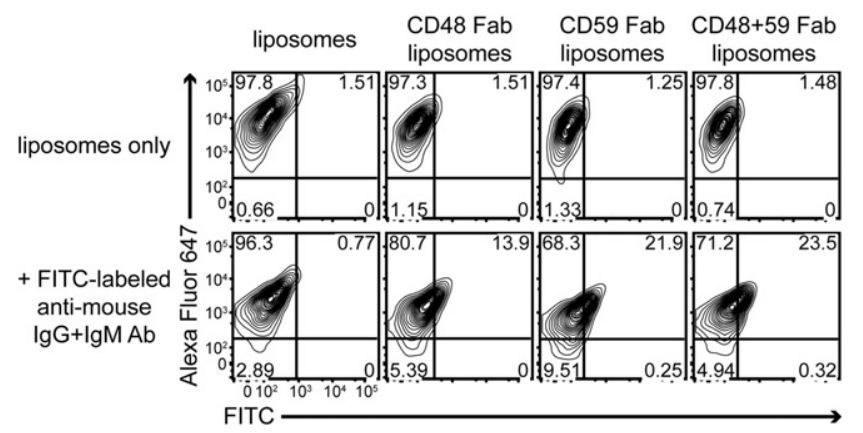

Figure 3. Fab antibody fragments are anchored in the membrane of AF647-loaded liposomes through the NDP and exposed on the liposomal surface. Control (empty) liposomes and liposomes harboring Fabs recognizing CD48, CD59 or both antigens were stained (or not) with FITC-labeled anti-mouse $\mathrm{IgG}+\mathrm{IgM}$ antibodies and analyzed by flow cytometry.

Region 1 (FR1) of $\mathrm{V}$ genes $^{21}$ in combination with the class-specific reverse primer targeting the end of the $\mathrm{CH} 1$ or the $\mathrm{CL}$ region, respectively, yielded several PCR products of the correct length (ca $650 \mathrm{bp}$; data not shown). In addition, the heavy chain of the CD48 mAb (of the IgG1 isotype) was amplified as a slightly longer product, terminating after the first cysteine-encoding codon at the beginning of the hinge region, encompassing aa 1-221. Subsequent sequencing and in silico analysis using IMGT/VQuest and IgBlast tools were used to identify and exclude aberrant VH-CH1 and VL-CL sequences that arose from unproductive rearrangement of the $\mathrm{V},(\mathrm{D}), \mathrm{J}$ alleles in the B cell or in the non-secreting myeloma fusion partner used for hybridoma production. ${ }^{22,23}$ Next, the productively rearranged heavy and light chain sequences were re-cloned into the Pichia expression vector pPICZ $\alpha \mathrm{A}$, combined into the single Fab expression vector encompassing both the heavy and the light chain expression cassettes (Supplementary Figure 1, $A$ ) and electroporated into the yeast Pichia pastoris.

Several zeocin-resistant Pichia clones with correctly genome-integrated cDNAs of both Fab chains were tested for the production of the Fab antibody fragments. By Western blotting, positive signals were detected when analyzing Pichia cell culture supernatants (data not shown) and the clones with the highest secretion of Fabs into the cell culture media were selected for large-scale cultivation and Fab purification. Subsequent analysis of purified Fabs by Western blotting (Figure 1) revealed that the CD48 Fab, the heavy chain of which encompassed the VH-CH1 region (designated as aa 1-216), showed altered migration at $\approx 70 \mathrm{kD}$ (Figure 1, $A$ right) under nonreducing conditions. This was due to an incorrectly folded heavy chain that predominantly migrated between 35 and $50 \mathrm{kD}$ as revealed by reducing SDS-PAGE and Western blotting with the anti-myc $\mathrm{mAb}$ (Figure 1, $B$ left). This finding was confirmed by treatment of the purified CD48 Fab (aa 1-216) with recombinant endoglycosidase F: the treated sample showed the same altered migration as the non-treated one (data not shown) indicating that the altered migration is not due to increased glycosylation but due to conformation. Nevertheless, this Fab variant proved functional in the flow cytometry analysis, because it stained positively human CD48-positive $\left(\mathrm{CD} 48^{+}\right)$Jurkat $\mathrm{T}$ cells but not mouse BW5147 thymoma cells (Figure 2, $A$ left). In contrast, the CD48 Fab variant with an additional cysteine in the heavy chain (designated as aa 1-221) showed improved folding, resulting in appearance of the $\approx 50 \mathrm{kD}$ band under non-reducing conditions (Figure 1, $A$ right), correlating with the increased proportion of the correctly folded heavy chain migrating at $\approx 30$ $\mathrm{kD}$ under reducing conditions (Figure 1, $B$ left). Increased folding resulted in improved binding to the $\mathrm{CD} 48^{+}$Jurkat cells, when $\approx 54 \%$ Jurkat cells scored positively comparing to $31 \%$ using the CD48 Fab (aa 1-216). For these reasons, the longer variant of the CD48 Fab was chosen for further studies. The CD59 Fab predominantly migrated at $\approx 50 \mathrm{kD}$ under non-reducing conditions (Figure 1, C) despite higher molecular weight bands of the heavy chain as revealed by Western blotting with the anti-myc mAb (Figure 1, D). It reacted only with the CD59 $9^{+}$Jurkat T cells but not with the BW5147 cells (Figure 2, B).

\section{Generation of Fab antibody fragments with the hydrophobic peptide}

To equip the Fab antibody fragments with the hydrophobic peptide derived from human SP-D (aa 242-248; QAAFSQY), we genetically fused the $\mathrm{C}$-terminus of the Fab heavy chains with a modified peptide and a linker with an $\mathrm{N} \rightarrow \mathrm{C}$ sequence DRDDQAAWFSQY that we called the neck domain peptide (NDP; Supplementary Figure 1, B). Previously, NDP was successfully used to anchor folate into the lipid bilayer of the liposomes without affecting liposomal integrity. ${ }^{9}$ Further, bioinformatic analysis of the NDP-heavy chain constructs revealed that NDP is extended away from the Fab variable and constant domains (Supplementary Figure 2), indicating that the Fab fragments should be efficiently inserted into the liposomal membrane via the C-terminal hydrophobic peptide, with the N-terminal antigen-binding sites well exposed. Indeed, introducing NDP into the Fab sequences had no detrimental effect onto the Fab folding (Figure 1, $A$ and $B$ ) or their functionality (Figure 2, $A$ right, 2, $B$ right). However, the direct comparison of the CD48 Fab-NDP and CD59 Fab-NDP constructs revealed higher affinity of the CD48 Fab-NDP to its respective antigen (Supplementary Figure 3 ) as well as its preferential recognition by our anti-mouse $\operatorname{IgG}+\operatorname{IgM}$ secondary reagent (Figure 2).

\section{Liposome functionalization with the Fab-NDP fragments}

Fab-functionalized $\mathrm{pH}$-sensitive liposomes were prepared by self-assembly when the dried lipid film was dispersed in PBS containing AF647 and the NDP-equipped Fabs specific to the CD48 or the CD59 molecule. Since both CD48 and CD59 reside in lipid rafts and were shown to associate with each other in human $\mathrm{T}$ cells, ${ }^{24}$ we also prepared bispecific liposomes harboring both CD48 and CD59 Fab fragments to learn whether these would preferentially target $\mathrm{CD} 48^{+} \mathrm{CD} 59^{+}$cells. Control liposomes were prepared without any additional protein, carrying only AF647 as a cargo. Based on the dynamic light scattering measurements (Table 1), all four preparations had a similar size of around $125-130 \mathrm{~nm}$ and a very narrow size 

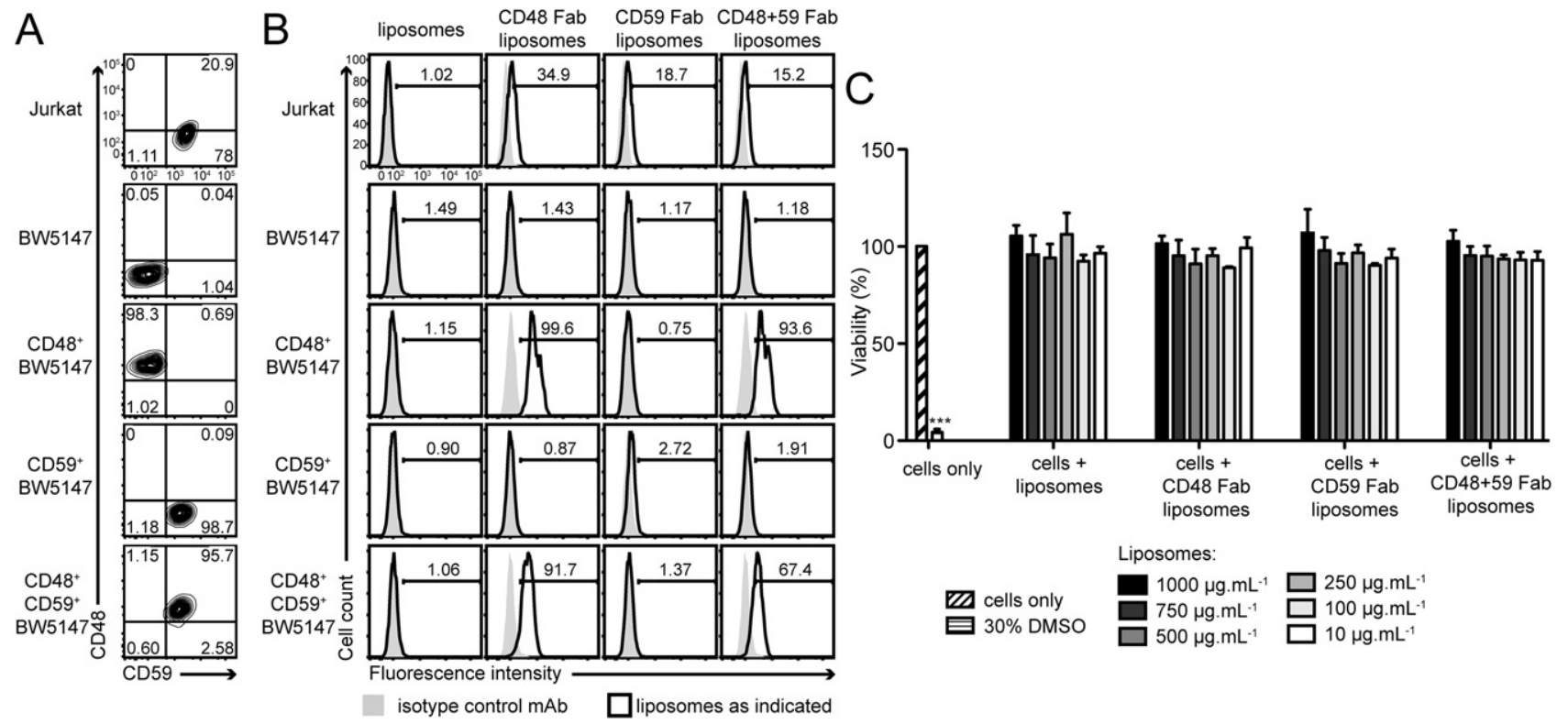

Figure 4. Liposomes with incorporated CD48 Fab, CD59 Fab or both Fabs are specifically binding to cells in an antigen-dependent manner, without cytotoxic effects. (A) Surface expression of the CD48 and CD59 molecules on Jurkat T cells and the mouse BW5147 cells (overexpressing either none, one or both antigens) was determined by flow cytometry following costaining with AF555-labeled CD48 mAb (MEM-102) and AF647-labeled CD59 mAb (MEM-43/5). (B) The indicated liposome samples were added to cells from (A) at $100 \mu \mathrm{g} \cdot \mathrm{mL}^{-1}$ for $30 \mathrm{~min}$ on ice. After washing the liposomal binding to the cells was visualized by FITC-labeled anti-mouse IgG + IgM antibodies and analyzed by flow cytometry. (C) To determine liposome cytotoxic effects, the viability of $\mathrm{CD} 48^{+} \mathrm{CD} 59^{+}$Jurkat $\mathrm{T}$ cells was assessed after $24 \mathrm{~h}$ contact with liposomal formulations and compared with the untreated (negative control) and $30 \%$ DMSO-treated cells (positive control). Data are mean \pm SEM from 3 experiments. ${ }^{* * *} p<0.001$, by one-way ANOVA.

distribution (polydispersity index $<0.05$ ), indicating that they were monodisperse. The $\zeta$ potentials of the different preparations were also similar and close to $0 \mathrm{mV}$. To test the presence of the Fabs on the liposomal surface, we incubated them with FITC-labeled anti-mouse $\operatorname{IgG}+\operatorname{IgM}$ antibodies and analyzed binding by flow cytometry. The anti-mouse antibodies recognized a significant proportion of the liposomes equipped with the Fabs but did not bind control liposomes (Figure 3). Also by ELISA, control liposomes were not recognized by the secondary antibody, while the Fab-functionalized liposomes contained 3.38 $\pm 0.50 \mu \mathrm{g} \mathrm{Fab} / 1 \mathrm{mg}$ liposome (for CD48 Fab liposomes), 3.76 $\pm 0.21 \mu \mathrm{g} \mathrm{Fab} / 1 \mathrm{mg}$ liposome (for CD59 Fab liposomes) or $4.18 \pm 1.72 \mu \mathrm{g} \mathrm{Fab} / 1 \mathrm{mg}$ liposome (for double-functionalized liposomes). Further, the flow cytometry analysis revealed that all preparations were highly loaded with the AF647 dye, though the Fab-equipped liposomes were loaded to a lesser degree than the control ones (Figure 3).

\section{Functional evaluation of the Fab-functionalized liposomes}

To verify the specificity of the prepared liposomal formulations, we used $\mathrm{CD} 48^{+} \mathrm{CD} 59^{+}$Jurkat T cells, negative BW5147 cells and also BW5147 cells transduced with either one or both antigens (Figure 4,A). The cells were incubated with the liposomes for $30 \mathrm{~min}$ on ice and liposomal binding to the cells was detected using FITC-labeled anti-mouse IgG + IgM antibodies (Figure 4, $B$ ). The Fab-functionalized liposomes correctly bound to the surface bearing the respective antigens:
CD48-functionalized liposomes reacted with $\mathrm{CD} 48^{+} \mathrm{CD} 59^{+}$ Jurkat and $\mathrm{CD} 48^{+} \mathrm{CD} 59^{-}$and $\mathrm{CD} 48^{+} \mathrm{CD} 59^{+}$BW5147 cells but not with $\mathrm{CD} 48^{-} \mathrm{CD} 59^{+}$or parental BW5147 cells. Conversely, the CD59-functionalized liposomes reacted only with double positive Jurkat and BW5147 cells and with CD48 $\mathrm{CD}^{2} 9^{+}$BW5147 cells. The bispecific liposomes reacted with all cells expressing either the CD48 or the CD59 molecule, though the binding to the $\mathrm{CD} 48^{-} \mathrm{CD} 59^{+}$BW5147 cells was weak. From these results we concluded that the bispecific liposomes predominantly bound through the CD48 Fab moiety that had shown higher affinity than the CD59 Fab (Figure 2, $A$ and $B$ and Supplementary Figure 3).

Since cytotoxicity is a major issue in nanodevice technology, ${ }_{1,25}$ we tested the prepared liposomal formulations with the $\mathrm{CD} 48^{+} \mathrm{CD} 59^{+}$Jurkat $\mathrm{T}$ cells. After $24 \mathrm{~h}$ we saw no detrimental effects on cell viability even when we incubated the cells with the high liposomal concentration of $1 \mathrm{mg} \cdot \mathrm{mL}^{-1}$ (Figure 4,C).

To scrutinize cellular uptake of our liposomes, we incubated the control and $\mathrm{CD} 48^{+} \mathrm{CD} 59^{+}$BW5147 cells with control or Fab-functionalized liposomes and counterstained them with anti-mouse AF488-labeled secondary antibodies on ice. Further steps were performed at $r$. t. to allow slow internalization of the liposomes. Confocal microscopy revealed that the Fab-functionalized liposomes bound to the surface of the $\mathrm{CD} 48^{+} \mathrm{CD} 59^{+}$but not to control BW5147 cells (Figure 5, $A$ ). They also appeared to readily release their cargo, the AF647 dye, 
A
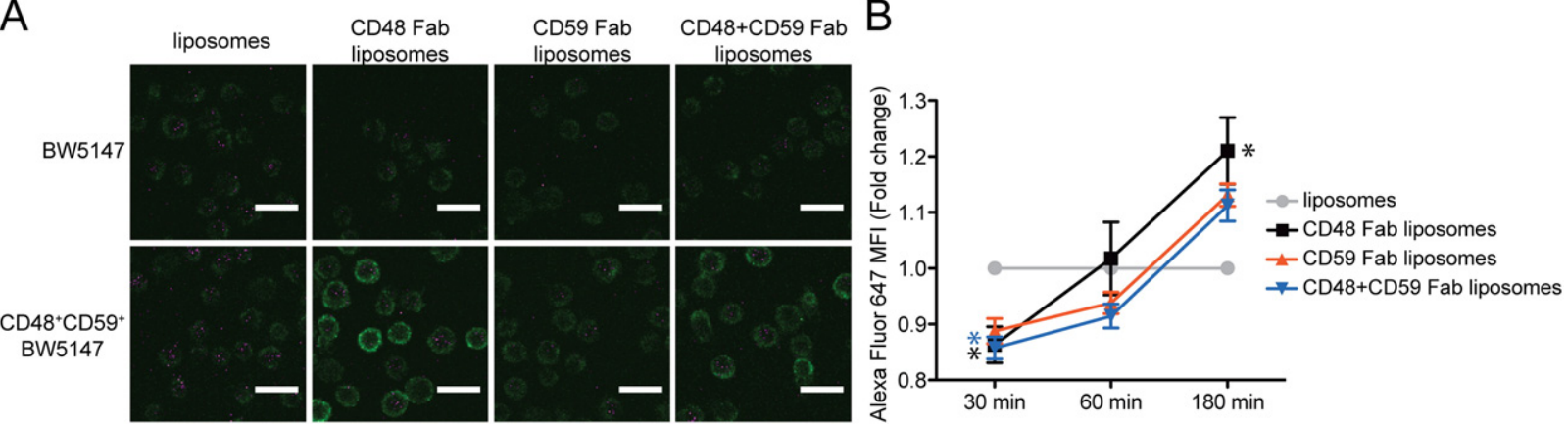

Figure 5. Liposomes with incorporated Fabs efficiently deliver AF647 into antigen-positive cells. (A) Confocal laser scanning microscopy images of control and $\mathrm{CD} 48^{+} \mathrm{CD} 59^{+}$BW5147 cells after incubation with the indicated AF647-loaded (in magenta) liposomes (100 $\mu \mathrm{g} \cdot \mathrm{mL}^{-1}$ for $30 \mu \mathrm{min}$ on ice) followed by immunostaining (30 min on ice) with FITC-labeled secondary antibodies (in green). Cells were washed, fixed and mounted at r. t. Scale bar represents $20 \mu \mathrm{m}$. (B) Liposome uptake by the control and $\mathrm{CD} 48^{+} \mathrm{CD} 59^{+} \mathrm{BW} 5147$ cells at $37^{\circ} \mathrm{C}$ over time was monitored by flow cytometry. In the graph, the ratio between the uptake by the $\mathrm{CD} 48^{+} \mathrm{CD} 59^{+}$BW5147 cells versus control BW5147 cells is shown. To account for the higher AF647 loading of the control liposomes (see Figure 3 ), the fluorescence of cells incubated with the Fab-functionalized liposomes was further normalized to fluorescence of the control liposome sample at each time point. Values represent mean \pm SEM of two experiments. ${ }^{*} p<0.05$, by one-way ANOVA.

into the target cells upon binding their respective antigen, because the target cells turned positive whereas the control BW5147 cells remained negative. AF647 staining of both cell types upon encountering of control liposomes was similar and higher than with the Fab-functionalized liposomes that might have been caused by the higher dye loading of the control liposomes (Figure 3). To quantify the specific uptake of the control and Fab-functionalized liposomes, we added them to the control and $\mathrm{CD} 48^{+} \mathrm{CD} 59^{+}$BW5147 cells and monitored the increase in AF647 fluorescence over time (Figure 5, B). To some degree, both cell types took in the cargo of all types of liposomes tested. Nevertheless, only the Fab-functionalized liposomes were preferentially taken up by the $\mathrm{CD} 48^{+} \mathrm{CD} 59^{+}$BW5147 cells (Figure 5, B) and fluorescence gradually increased over time. The most robust specific uptake was detected with the CD48 Fab-functionalized liposomes, which was in line with their highest reactivity in the cell surface binding assay (Figure 4, B).

Additionally, we made a larger scale preparation of control and CD48 Fab-functionalized liposomes that were equally loaded with AF647 and also not cytotoxic (Supplementary Figure 4, $A$ and $B$ ). We thoroughly tested them using an array of human CD48 molecule positive (human Raji B cells, human Jurkat T cells, mouse BW5147 thymoma cells expressing the human CD48 molecule) and human CD48 molecule negative (mouse thymoma BW5147 cells, human myeloerythroid K-562 cells) cell lines, and observed the preferential uptake of the CD48 Fab-functionalized liposomes only by the CD $48^{+}$cells (Supplementary Figure 4,C). Nevertheless, a basal liposomal uptake over time was detectable with each cell line that was higher than the uptake of free AF647.

Lastly, we tested the CD48 Fab liposomes in vivo for their ability to specifically recognize $\mathrm{CD} 48^{+}$Raji B cell tumor xenografts implanted on the chest of nude mice. Strikingly, we observed the preferential AF647 accumulation in the tumor area only when CD48 Fab-functionalized liposomes, but not control liposomes were injected intravenously via the tail vein (Figure 6, A and $B$ ). In one mouse where the tumor had grown to a size of only $20 \mathrm{~mm}^{3}$, the CD48 Fab liposomes also recognized the axillary lymph nodes (right mouse in Figure 6, $A$ ). This was because the Raji B cells disseminated there as found by analysis of the lymph nodes using a human CD19 mAb.

\section{Discussion}

In this study, we have successfully developed a method to convert mAbs into recombinant Fabs and efficiently produced them in P. pastoris. P. pastoris is a popular host for heterologous protein expression for several reasons: cost effectiveness, easy genetic manipulation, availability of various vectors that allow tightly regulated expression from inducible promoters and especially production of high levels of correctly folded heterologous proteins that contain correct disulfide bonds, are proteolytically processed and glycosylated, similarly to mammalian proteins. ${ }^{26,27}$ While the production of $\mathrm{scFv}$ fragments in $P$. pastoris was easily achieved, ${ }^{28}$ previous studies have shown that Fab expression was more challenging and observed only in the weaker constitutive expression system driven by the $\mathrm{p} G A P$ promoter but not in the strong methanol-inducible system that we used. $^{29}$ In our hands, both native Fabs and Fabs equipped with the neck domain peptide (NDP) on the C-terminus of the Fab heavy chain expressed well and were highly functional.

We previously demonstrated that the hydrophobic NPD inserts deeply into the lipid bilayer and anchors folate onto liposomal surface. ${ }^{9}$ Also others have used hydrophobic peptides derived from bacteriophages and genetically fused them to targeting peptides to functionalize liposomes. ${ }^{30,31}$ Now we show that NDP anchors onto liposomes without the need of harmful cross-linkers not only small ligands but also Fabs with the antigen-binding site well exposed. The resulting liposomes, as demonstrated by the CD48- and CD59-Fab liposomes and double-specific liposomes containing both Fabs, are highly specific to the respective antigen-positive cells. Furthermore, these liposomes are able to efficiently deliver the liposome cargo, the AF647 dye, into the antigen-positive cells, both in vitro and in vivo. This may be due to the fact that the CD59 and CD48 


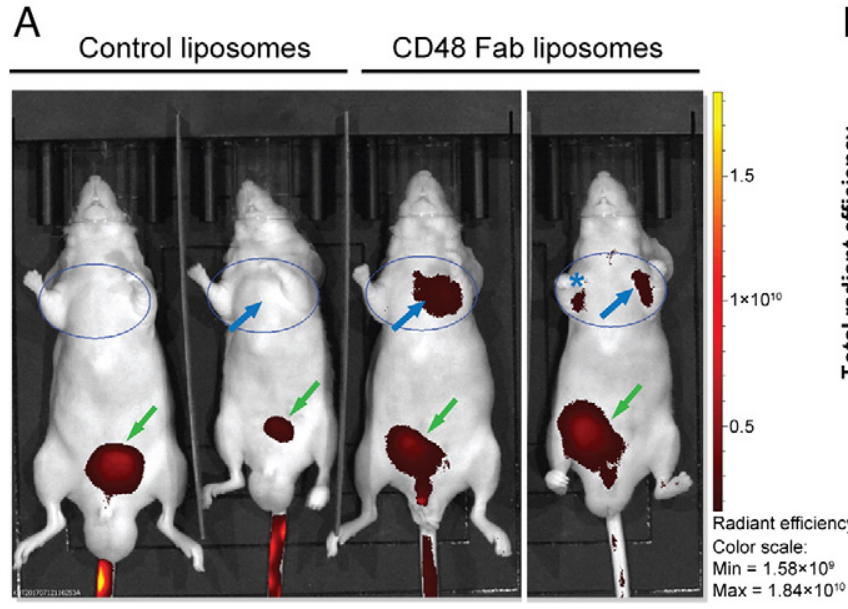

B

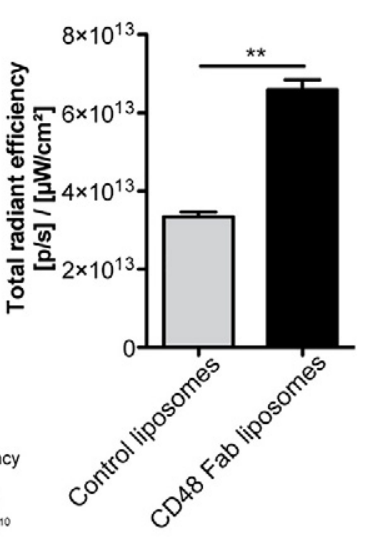

Figure 6. Liposomes with incorporated CD48 Fab efficiently target CD48 ${ }^{+}$Raji tumor xenografts. (A) AF647-labeled CD48 Fab- or control liposomes were injected into nude mice with subcutaneously implanted Raji cells on the chest. Thirty minutes later we imaged the mice using an in vivo imager. Red-to-yellow scale shows the intensity of AF647 fluorescence, blue ovals depict the areas that were quantified in (B) and blue arrows indicate measurable tumors. CD48 Fab liposomes targeted either tumor mass or axillary lymph nodes (blue asterisk) with disseminated Raji cells. Additionally, we observed AF647 accumulation in the bladder of all mice (green arrows), an indication of the fast renal clearance. (B) Quantification of AF647 fluorescence on the chest of mice injected with either control or CD48 Fab liposomes. Values represent mean \pm SEM. ${ }^{* *} p<0.01$, by unpaired two-tailed $t$ test.

molecules, similarly to other GPI-anchored molecules, easily internalize through the clathrin-independent mechanism. ${ }^{32}$ Previous studies showed that therapeutic efficacy of antibody-targeted liposomal drugs was improved when the liposomes targeted internalizing epitopes. For instance, doxorubicin-loaded CD19 mAb-conjugated liposomes targeting the internalizing B cell-specific molecule CD19 showed higher degree of internalization and cytotoxicity in human B-lymphoma cells than doxorubicin-loaded CD20 mAb-conjugated liposomes against the non-internalizing molecule $\mathrm{CD} 20 .{ }^{33}$

In conclusion, the use of liposomes, decorated with a hydrophobic peptide-anchored antibody fragment of choice against an internalizing antigen, and loaded with a therapeutic drug, opens new avenues for efficient treatment of various human diseases.

\section{Appendix A. Supplementary data}

Supplementary data to this article can be found online at https://doi.org/10.1016/j.nano.2017.09.003.

\section{References}

1. Sanvicens N, Marco MP. Multifunctional nanoparticles - properties and prospects for their use in human medicine. Trends Biotechnol 2008;26:425-33.

2. Sawant RR, Torchilin VP. Challenges in development of targeted liposomal therapeutics. AAPS J 2012;14:303-15.

3. Noble GT, Stefanick JF, Ashley JD, Kiziltepe T, Bilgicer B. Ligandtargeted liposome design: challenges and fundamental considerations. Trends Biotechnol 2014;32:32-45.

4. Bregoli L, Movia D, Gavigan-Imedio JD, Lysaght J, Reynolds J, PrinaMello A. Nanomedicine applied to translational oncology: a future perspective on cancer treatment. Nanomedicine 2016;12:81-103.
5. Wang R, Xiao R, Zeng Z, Xu L, Wang J. Application of poly(ethylene glycol)-distearoylphosphatidylethanolamine (PEG-DSPE) block copolymers and their derivatives as nanomaterials in drug delivery. Int $J$ Nanomedicine 2012;7:4185-98.

6. Nobs L, Buchegger F, Gurny R, Allemann E. Current methods for attaching targeting ligands to liposomes and nanoparticles. J Pharm Sci 2004;93:1980-92.

7. Botas C, Poulain F, Akiyama J, Brown C, Allen L, Goerke J, et al. Altered surfactant homeostasis and alveolar type II cell morphology in mice lacking surfactant protein D. Proc Natl Acad Sci USA 1998;95:11869-74.

8. Kishore U, Wang JY, Hoppe HJ, Reid KB. The alpha-helical neck region of human lung surfactant protein D is essential for the binding of the carbohydrate recognition domains to lipopolysaccharides and phospholipids. Biochem J 1996;318(Pt 2):505-11.

9. Nogueira E, Mangialavori IC, Loureiro A, Azoia NG, Saarria MP, Nogueira P, et al. Peptide anchor for folate-targeted liposomal delivery. Biomacromolecules 2015;16:2904-10.

10. Nogueira E, Lager F, Le Roux D, Nogueira P, Freitas J, Charvet C, et al. Enhancing methotrexate tolerance with folate tagged liposomes in arthritic mice. J Biomed Nanotechnol 2015;11:2243-52.

11. Horejsi V. The roles of membrane microdomains (rafts) in $\mathrm{T}$ cell activation. Immunol Rev 2003;191:148-64.

12. Meri S, Morgan BP, Davies A, Daniels RH, Olavesen MG, Waldmann H, et al. Human protectin (CD59), an 18,000-20,000 MW complement lysis restricting factor, inhibits C5b-8 catalysed insertion of C9 into lipid bilayers. Immunology 1990;71:1-9.

13. McArdel SL, Terhorst C, Sharpe AH. Roles of CD48 in regulating immunity and tolerance. Clin Immunol 2016;164:10-20.

14. Stefanova I, Horejsi V, Ansotegui IJ, Knapp W, Stockinger H. GPIanchored cell-surface molecules complexed to protein tyrosine kinases. Science 1991;254:1016-9.

15. Korty PE, Brando C, Shevach EM. CD59 functions as a signaltransducing molecule for human $\mathrm{T}$ cell activation. J Immunol 1991;146:4092-8.

16. Stulnig TM, Berger M, Sigmund T, Stockinger H, Horejsi V, Waldhausl W. Signal transduction via glycosyl phosphatidylinositol-anchored proteins in T cells is inhibited by lowering cellular cholesterol. $J$ Biol Chem 1997;272:19242-7. 
17. Kato K, Koyanagi M, Okada H, Takanashi T, Wong YW, Williams AF, et al. CD48 is a counter-receptor for mouse CD2 and is involved in T cell activation. $J$ Exp Med 1992;176:1241-9.

18. Muhammad A, Schiller HB, Forster F, Eckerstorfer P, Geyeregger R, Leksa V, et al. Sequential cooperation of CD2 and CD48 in the buildup of the early TCR signalosome. J Immunol 2009;182:7672-80.

19. Lipp AM, Juhasz K, Paar C, Ogris C, Eckerstorfer P, Thuenauer R, et al. Lck mediates signal transmission from CD59 to the TCR/CD3 pathway in Jurkat T cells. PLoS One 2014;9:e85934.

20. Drbal K, Moertelmaier M, Holzhauser C, Muhammad A, Fuertbauer E, Howorka S, et al. Single-molecule microscopy reveals heterogeneous dynamics of lipid raft components upon TCR engagement. Int Immunol 2007;19:675-84.

21. Amersdorfer P, Wong C, Chen S, Smith T, Deshpande S, Sheridan R, et al. Molecular characterization of murine humoral immune response to botulinum neurotoxin type a binding domain as assessed by using phage antibody libraries. Infect Immun 1997;65:3743-52.

22. Vidarsson G, van de Winkel JG, van Dijk MA. Multiplex screening for functionally rearranged immunoglobulin variable regions reveals expression of hybridoma-specific aberrant V-genes. J Immunol Methods 2001;249:245-52.

23. Irani Y, Tea M, Tilton RG, Coster DJ, Williams KA, Brereton HM. PCR amplification of the functional immunoglobulin heavy chain variable gene from a hybridoma in the presence of two aberrant transcripts. J Immunol Methods 2008;336:246-50.
24. Cinek T, Hilgert I, Horejsi V. An alternative way of CD4 and CD8 association with protein kinases of the Src family. Immunogenetics 1995;41:110-6.

25. Onoue S, Yamada S, Chan HK. Nanodrugs: pharmacokinetics and safety. Nanomedicine 2014;9:1025-37.

26. Cregg JM. Introduction: distinctions between Pichia pastoris and other expression systems. Methods Mol Biol 2007;389:1-10.

27. Bollok M, Resina D, Valero F, Ferrer P. Recent patents on the Pichia pastoris expression system: expanding the toolbox for recombinant protein production. Recent Pat Biotechnol 2009;3:192-201.

28. Ridder R, Schmitz R, Legay F, Gram H. Generation of rabbit monoclonal antibody fragments from a combinatorial phage display library and their production in the yeast Pichia pastoris. Biotechnology (N Y) 1995;13:255-60.

29. Gasser B, Maurer M, Gach J, Kunert R, Mattanovich D. Engineering of Pichia pastoris for improved production of antibody fragments. Biotechnol Bioeng 2006;94:353-61.

30. Jayanna PK, Torchilin VP, Petrenko VA. Liposomes targeted by fusion phage proteins. Nanomedicine 2009;5:83-9.

31. Bedi D, Musacchio T, Fagbohun OA, Gillespie JW, Deinnocentes P, Bird RC, et al. Delivery of siRNA into breast cancer cells via phage fusion protein-targeted liposomes. Nanomedicine 2011;7:315-23.

32. Sabharanjak S, Sharma P, Parton RG, Mayor S. GPI-anchored proteins are delivered to recycling endosomes via a distinct cdc42-regulated, clathrin-independent pinocytic pathway. Dev Cell 2002;2:411-23.

33. Sapra $P$, Allen TM. Internalizing antibodies are necessary for improved therapeutic efficacy of antibody-targeted liposomal drugs. Cancer Res 2002;62:7190-4. 